\title{
Chondroblastic osteosarcoma of maxilla: a case report and review of literature
}

Received: 18 February 2009 / Accepted: 12 June 2009

(C) Association of Oral and Maxillofacial Surgeons of India 2009

\begin{abstract}
Craniofacial osteosarcoma is a relatively rare entity comprising about $6.5-7 \%$ of all osteosarcomas, whereas it is reported to be one of the most common malignancies of long bones. We present a case of chondroblastic variant of osteosarcoma (COS) of the maxilla in an 18 year-old-girl. She was treated with a subtotal maxillectomy and later referred for adjuvant chemotherapy. The relevant review of literature with regard to clinical, radiographic findings, histopathological similarities to Chondrosarcoma (CS), role of Immunohistochemistry (IHC) and management modalities of Osteosarcoma of jaw bones (OSJ) in comparison with Osteosarcoma of the long bones (OSL) are being presented in this report.
\end{abstract}

Keywords Osteosarcoma $\cdot$ Bone neoplasm $\cdot$ Maxilla

Introduction

Osteosarcoma is an aggressive primary bone tumor composed of connective tissue cells directly producing osteoid and bone [1]. Although it is the most common malignancy of long bones after multiple myeloma [2], it is a relatively rarer entity in the craniofacial region. Craniofacial osteosarcomas constitute only about $6.5-$ $7 \%$ of all osteosarcomas [3]. Despite sharing common histopathological features, Osteosarcoma of Jaw bones (OSJ) and Osteosarcoma of Long bones (OSL) are distinct biological entities. This article is presented to share our experience with a case of osteosarcoma of maxilla in a young girl and to review the relevant literature regarding OSJ as a distinct clinical entity in contrast with OSL.

\section{Case report}

An 18-year-old female patient presented to the department of Oral and Maxillofacial surgery with the chief complaint of a slow growing asymptomatic swelling in the left upper buccal vestibule since 2 months. She had no significant medical history but gave a significant family history. Her maternal grandfather succumbed to hepatic carcinoma and her mother succumbed to carcinoma breast.

On examination the patient was apparently healthy except for a mild swelling on the left side of her cheek. No cervical lymphadenopathy was evident, and all the cranial nerves were normal.

The mouth opening was adequate. Intraorally a solitary well circumscribed roughly oval to round swelling measuring $3 \times 3$ $\mathrm{cm}$ in diameter was located in the left upper buccal vestibule extending from the distal aspect of canine till the mesial aspect of the second molar. The swelling obliterated the entire buccal vestibule and had a mild palatal extension in the region of the premolars. All the teeth in the quadrant were clinically normal and vital. It was a well demarcated swelling with normal appearing overlying mucosa. There was no evidence of nasal obstruction or ophthalmologic signs of extension of the lesions into these anatomical regions. There were also no signs of neurosensory deficit associated with the infraorbital nerve. On palpation it was non tender, bony hard in consistency and fixed to the underlying structures.

An orthopantomogram, paranasal sinus view, maxillary occlusal view and an intraoral periapical view were taken. The radiographs revealed poorly defined mixed radiolucentradioopaque lesion in the left maxillary
Kumaravelu $C^{1} \cdot$ Sathya Kumar $D^{2}$. Chitra Chakravarthy' ${ }^{2}$ Kishore Kumar $\mathrm{RV}^{3} \cdot$ Rajasekhar Gali $^{4} \otimes \cdot$ Gokul Chandra Reddy $\mathbf{M}^{2} \cdot$ Sridhar Reddy $\mathrm{K}^{4}$

${ }^{1}$ Professor \& Head

${ }^{2}$ Professor

${ }^{3}$ Reader

${ }^{4}$ Senior Lecturer

Dept. of Oral and Maxillofacial Surgery, Narayana Dental College and Hospital, Andhra Pradesh

Address for correspondence:

Rajasekhar Gali

Dept. of Oral and Maxillofacial Surgery

Narayana Dental College and Hospital

Nellore-524002

Andhra Pradesh, India

E-mail: dr_rajasekhar_g@yahoo.com

alveolus extending into the floor of the maxillary antrum, with uniform widening of the periodontal ligament space in relation to second premolar and first molar.

A CT scan of the paranasal sinus region showed a $3 \times 3 \mathrm{~cm}$ osteosclerotic and lytic lesion extending from alveolar process of the left maxilla causing resorption of the lateral wall and floor of the maxillary antrum and encroaching into it. The lesion also showed dense foci calcifications within it. Her hematological and biochemical profile including serum alkaline phosphatase were normal.

After a negative aspiration, an incisional biopsy was done under local anesthesia from the left buccal vestibule. The buccal cortical plate in relation to the swelling was found to be completely resorbed and a solid mass was seen in the submucosal plane.

The histopathology report revealed hyaline type of cartilagenous tissue with varying cellularity. Many mildly pleomorphic round/oval cells with dispersed chromatin, prominent nucleoli enclosed in lacunae were seen in haphazardly arranged sheets. Scattered binucleated and multinucleated cells and foci of calcification and endochondral ossification were evident. Mitotic activity was seen with $1-2 / 5 \mathrm{HpF}$. The picture was suggestive of a chondroblastic variant of osteosarcoma. As extensively 
Table 1 Showing the differences between OSL \& OSJ

\begin{tabular}{|c|c|c|}
\hline Features & Osteosarcoma - long bones & Osteosarcoma - jaw bones \\
\hline 1. Age of occurrence & $\begin{array}{l}\text { Bimodal distribution adolescent age } \\
\text { group and 5th decade }[1,11]\end{array}$ & $3 \mathrm{rd} / 4$ th decade $[11,14]$ \\
\hline $\begin{array}{l}\text { 2. Evidence of subclinical micro } \\
\text { metastasis at the time of initial diagnosis }\end{array}$ & $80 \%[1]$ & $18 \%[2,14]$ \\
\hline 3. Site of occurrence & $\begin{array}{l}\text { Distal femoral metaphysis proximal tibia } \\
\text { and humeral metaphysis [1] }\end{array}$ & $\begin{array}{l}\text { Maxilla: anterior alveolus and antrum } \\
\text { Mandible: Body, symphysis and } \\
\text { ascending ramus }[4,6]\end{array}$ \\
\hline 4. Common sites of metastasis & Lungs, bones and kidneys[1] & Lung brain $[2,14]$ \\
\hline 5. Most common cause of mortality & Distant metastasis [1] & Locoregional recurrence $[2,14]$ \\
\hline 6. Management protocols & Surgery + adjuvant chemotherapy [15] & $\begin{array}{l}\text { Radical Surgery-(main stay) }+/ \text { - adjuvant } \\
\text { chemotherapy [17] (developed for OSL) }\end{array}$ \\
\hline 7. Role of growth spurt & Related to growth spurt [11] & Not related to growth spurt [11] \\
\hline 8. Clinical feature at presentation & Painful swelling [1] & Painless swelling-first sign [5] \\
\hline 9. Neuroparesthesia & Rare [1] & Paresthesia present [5] \\
\hline 10. Prominent veins and distal edema & Positive [11] & Negative [11] \\
\hline
\end{tabular}

cellular chondroid areas were seen it was difficult to exclude the possibility of a chondrosarcoma. An immunohistochemical study was done to differentiate between CS and COS. It was found to be positive for Vimentin and Epithelial Membrane Antigen (EMA) and negative for $\mathrm{S} 100$ and cytokeratin-19. These reports confirmed the diagnosis of chondroblastic variant of osteosarcoma.

Possibility of metastasis to the brain and lungs was ruled out after an MRI brain and CT thorax.

The patient was taken up for surgery; subtotal inferior maxillectomy on the left side through transoral approach was done with clearance margins of $1.5 \mathrm{~cm}$ around the lesion. The resultant antral defect was lined by a split skin graft harvested from right thigh and an interim obturator was placed. The postoperative period was uneventful and the patient was discharged on the 5th day.

One month postoperatively the interim obturator was replaced by a new acrylic obturator with a soft liner. She was then referred for chemotherapy and was advised periodical check up.

\section{Discussion}

Osteosarcoma (OS) is a tumor composed of malignant connective tissue cells, directly producing osteoid and bone [1]. Osteosarcoma is the most common primary malignancy of bone after multiple myeloma and makes upto about $20 \%$ of all the sarcomas of the skeleton [2]. The most common site of occurrence is the distal femoral metaphysis followed by the proximal tibia and humeral metaphysis [1].

Craniofacial osteosarcoma is a relatively rarer entity and osteosarcomas of the jaws (OSJ) constitute only about $6.5-7 \%$ of all osteosarcomas [3]. OSJ differs from OS of long bones in its biological behaviour, though they share common histological features. Table 1 summarizes the differences between the two entities. The average age of onset of jaw lesions is in the 4 th decade, with a mean age of 34 years, but cases have been reported in patients of all ages. The common presenting feature is a rapidly growing swelling with localized pain [4]. August et al. [5] in a study of 30 patients with OSJ reported that the most common presenting symptom was swelling without pain. Other signs and symptoms include displacement and loosening of teeth, paresthesia, epistaxis and nasal obstruction.

The average duration of symptoms before diagnosis is $3-4$ months. The most frequent locations in the craniofacial region are alveolar ridge and antrum in the maxilla, the body, symphysis and ascending ramus in the mandible [4,6].

The radiographic appearance of OSJ depends on the interaction of three factors: bone destruction, bone production with mineralization and periosteal new bone formation. Lesions can thus appear radiolucent, radiolucent with fluffy cloud-like radio opaque areas or entirely radio opaque. The most common presentation is a mixed radiolucency with radio opaque pattern and poorly defined irregular borders [7]. The tumor commonly perforates the cortical plates and extends into the soft tissue as seen in our case. A classical sunray/sunburst radiographic pattern has been described for OS. This occurs due to the periosteal reaction producing bone perpendicular to bone surface. This presentation is not pathognomonic as it can be seen with other neoplastic and reactive lesions also. Other radiographic findings include root resorption and a symmetrical widening of the periodontal ligament space around the teeth in the area of the lesion as seen in our case. CT and MRI are superior diagnostic tools, especially to detect intramedullary and extramedullary involvement, tumor calcification and invasion into adjacent tissues, particularly pterygopalatine fossa, infra temporal fossa and cranial cavity [7,8]. Nakayama et al. [9] proposed a classification of CT pattern found in OSJ based on osteogenesis and signs of bone destruction. He proposed 4 types which were osteogenic with or without bone destruction and osteolytic with or without bone destruction. He reported better prognosis for osteogenic type without bone destruction. Our case falls into the group of osteogenic without bone destruction.

Histologically the diagnosis of OS is made when sarcomatous stroma is seen to directly elaborate osteoid or primitive bone [7]. The predominant histological variants described are osteoblastic, fibroblastic and chondroblastic varieties [10]. The jaw lesions are predominantly chondroblastic [2,3]. 


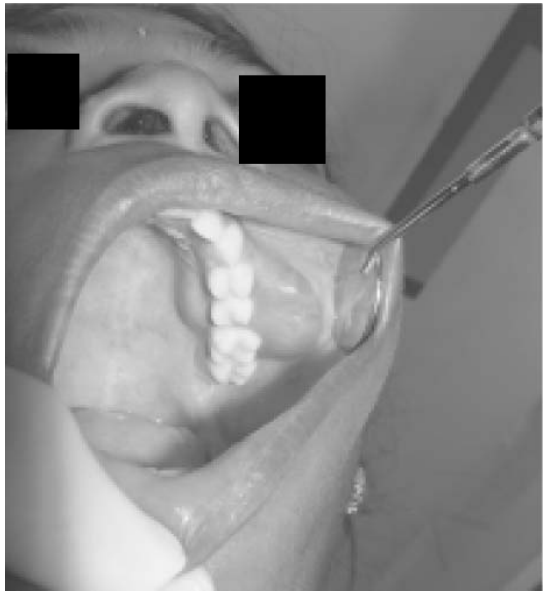

Fig. 1 Preoperative view

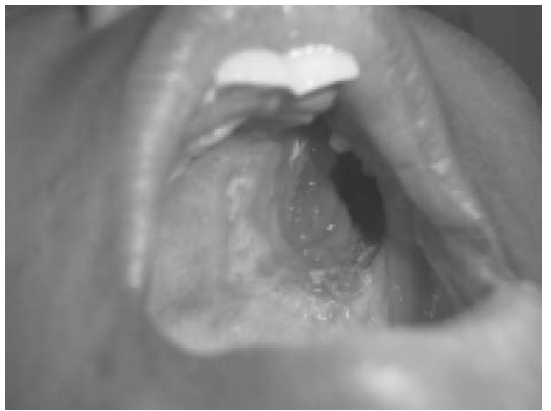

Fig. 46 months postoperative view

Although histopathological subtypes have not been shown to have prognostic significance [4], Broder's grading of the tumor based on the degree of cellular anaplasia is prognostically significant. High grade OSJ has been reported to be always associated with poorer prognosis [11]. Other histopathological features include cellular pleomorphism, hyperchromatic nuclei, bizarre mitosis, multinucleated giant cells, malignant osteoblasts with or without malignant cartilage which may be the predominant component in few lesions.

The presence of predominant cartilaginous component presents a diagnostic dilemma to the pathologist. Nusret Akpolat et al. [12] reported a case of sacral OS in which the FNAC showed features of a chordoma, incisional biopsy revealed a Chondrosarcoma (CS) and finally the total resected specimen reported a chondroblastic variant of osteosarcoma (COS). COS can be confused with chondrosarcoma in small biopsy specimens and differential diagnosis can be difficult. Osteoid formation with in the tumor is the most important histomorphologic diagnostic feature. It is not

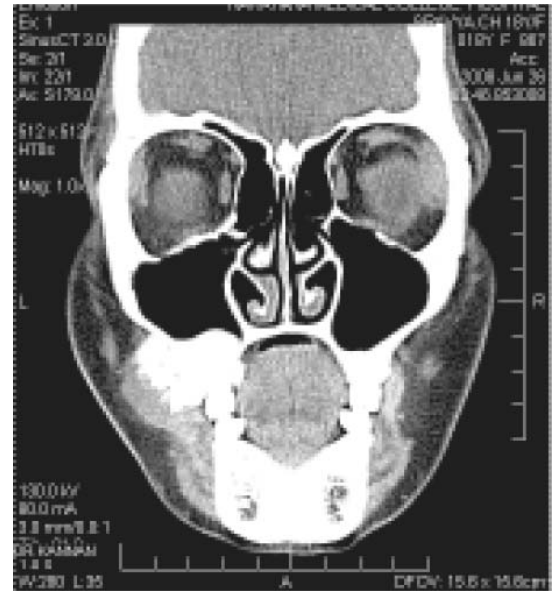

Fig. 2 CT scan showing the lesion involving the floor of left maxillary sinus

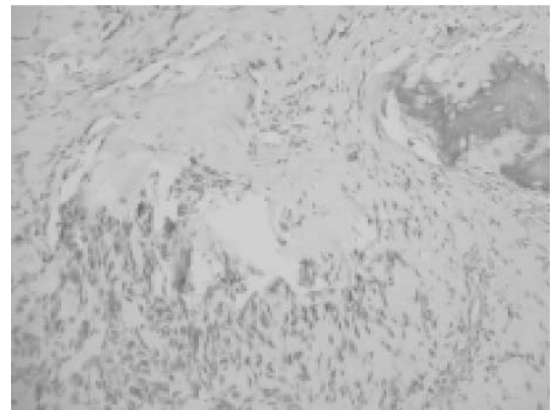

Fig. 5 Low power magnification (100x) showing spindle shaped tumor cells with hyperchromatic nuclei, mononuclear tumor giant cells and tumor osteoid showing basophilic staining

always possible to visualise osteoid in the small biopsy specimens. As a consequence COS may be misdiagnosed as CS on incisional biopsy as happened in our case. False diagnosis rate has been reported to be as high as $44 \%$ [12]. Immunohistochemistry (IHC) plays an important role in the differentiation between CS and COS.

IHC will show CS to be positive for S100 and Vimentin and negative for Cytokeratin and EMA (Epithelial Membrane Antigen). COS will be positive for Vimentin, EMA, S100 and rarely Cytokeratin [12]. The IHC profile of our patient was Vimentin and EMA positive; S100 and Cytokeratin 19 negative. Unni et al. [13] emphasised that in an adolescent patient in early adult hood, if the tumor morphologically constitutes a neoplastic cartilage and with no osteoid formation it must be considered and treated as COS unless proven to be otherwise. CS is predominantly seen in the elderly (4th6th decade) and is rare in adolescents and early adults. The need to differentiate between these two entities exists, because the prognosis of CS is better and metastatic tendency is lower.

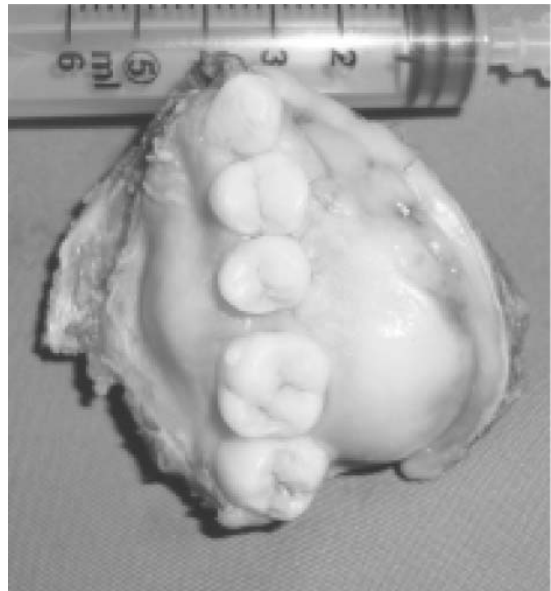

Fig. 3 Resected specimen

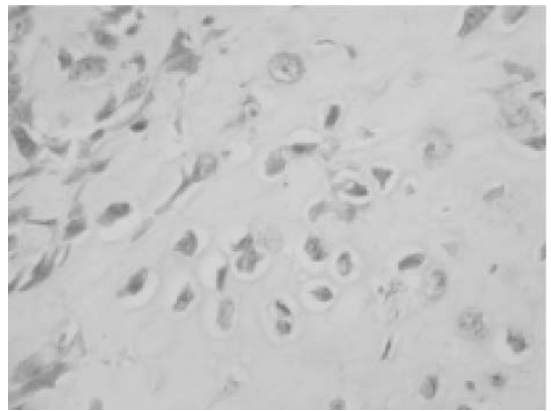

Fig. 6 High power magnification (400X) showing vesicular nuclei with prominent nucleoli, lacunae containing chondroblasts

OS of long bones metastasizes by hematogenous route than by lymphatic vessels. At the time of diagnosis grossly apparent metastatic disease is present in $10-$ $20 \%$ of all patients. At autopsy the most frequent sites of metastasis were found to be lungs, bones and kidneys [1]. OSJ does not metastasize as often as extragnathic lesions. The most common sites are lungs and brain [2,14].

Radical surgery was the main stay of treatment for OS of long bones, prior to the advent of adjuvant chemotherapy. In 1993 Cooperative Osteosarcoma Study group (COSS) reported on the use of high dose of methotrexate, doxorubicin and cisplatinum for adjuvant chemotherapy in osteosarcoma of long bones. They achieved 8 year metastasis free survival of $60-70 \%$ [15]. Radiation therapy appears to have no significant benefit.

For OSJ, radical surgery with clearance margins of $1.5-2 \mathrm{~cm}$ is still the main stay of treatment. Lesions of maxilla are generally approached through lateral rhinotomy with lip split incision with or without lynch extension, depending on extent of the lesion. 
A strict transoral route can also be used for smaller lesions. Partial or total maxillectomy with or without orbital exenteration is generally done as the clinical situation demands.

Pogrel et al. [16] have described a subtotal inferior maxillectomy for selected malignancies located on the alveolar ridge, palate and involving the antral floor.

Results with the use of adjuvant chemotherapy along with surgery for OSJ had been variable. Smeele et al. [17] investigated the value of chemotherapy in the treatment of craniofacial osteosarcoma by analyzing 201 reviewed cases. They found that the over all and disease free survival rates significantly improved with chemotherapy. Raymond et al. [18] reported 33\% 5 year survival for patients treated with adjuvant chemotherapy and surgery and $41 \% 5$ year disease free survival for those treated with surgery alone; the difference was not significant. Chemotherapeutic protocols differ in agents used, dosages, and intervals. The differences in protocol make it difficult to draw conclusions about their efficacy. August et al. [5] reported 30 patients with OSJ treated with combinations of surgery, radiation therapy and chemotherapy. Factors associated with statistically significant poorer prognosis include neurosensory deficit as the presenting symptom, increasing age of patients and surgical margins of less than $5 \mathrm{~mm}$. Patients receiving chemotherapy with 4 or more agents had better prognosis. In summary radical surgical resection combined with adjuvant chemotherapy regimens developed for long bones are being applied in the treatment of OSJ in most of the medical centres world over.

Another important point worth mentioning is the histopathological resemblance of chondroblastic osteosarcoma to chondrosarcoma.

The presence of predominant cartilaginous component presents a diagnostic dilemma to the pathologist. Nusret Akpolat et al. [12] reported a case of sacral OS in which the FNAC showed features of a chordoma, incisional biopsy revealed a chondrosarcoma and finally the total resected specimen was reported to be a chondroblastic variant of osteosarcoma . Chondroblastic variant of osteosarcoma can be confused with chondrosarcoma in small biopsy specimens and differential diagnosis can be difficult. Osteoid formation within the tumor is the most important histomorphologic diagnostic feature. But it is not always possible to visualize osteoid in the small biopsy specimens.

As a consequence COS may be misdiagnosed as CS on incisional biopsy as it happened in our case. False diagnosis rate has been reported to be as high as $44 \%$ [12]. Immunohistochemistry plays an important role in the differentiation between CS and COS. Immunohistochemistry will show CS to be positive for S100 and Vimentin and negative for cytokeratin and Epithelial Membrane Antigen (EMA). COS will be positive for vimentin, EMA, S100 and rarely Cytokeratin [12]. The IHC profile of our patient was Vimentin and EMA positive; S100 and Cytokeratin19 negative. Unni et al. [13] emphasized that in an adolescent patient in early adulthood, if the tumor morphologically constitutes a neoplastic cartilage with no osteoid formation it must still be considered and treated as COS unless proven to be otherwise. CS is predominantly seen in the elderly (4th-6th decade) and is rare in adolescents and early adults. The need to differentiate between these two entities exists, because the prognosis of CS is better and metastatic tendency is lower.

\section{Conclusion}

Chondroblastic osteosarcoma is a histopathological diagnosis. It is usually confused with the presentation of a chondrosarcoma. IHC in our case proved to be useful in the confirmation of the diagnosis. Although the histopathological picture, of osteosarcoma of long bones and jaw bones exhibit similarity, their management modalities may not necessarily be the same. Since loco-regional recurrence is the main cause of mortality in OSJ, meticulous wide resection seems to be the mainstay of treatment. Surgery followed by chemotherapy is followed by most centres around the world. As chemotherapy regimens for OSJ are not standardized world over, it is difficult to specifically attribute it's effectiveness in the management of OSJ.

\section{References}

1. Huvos AG (1991) Osteogenic sarcoma.in bone tumors: Diagnosis, treatment and prognosis, ed.2. Philadelphia, W.B.Saunders, $85-116$

2. Zarbo RJ (1993) Malignant non odontogenic neoplasms of the jaws. In Regezi JA, Sciubba.J(eds) : Oral pathology : Clinical- pathologic correlations, ed 2. Philadelphia, WB Saunders, 436-442

3. Garrington GE, Scofield HH, Cornyn J, Hooker SP (1967) Osteosarcoma of the jaws: Analysis of 56 cases. Cancer 20(3): 377-391
4. Clark J, Unni KK, Dahlin DC, Devine KD (1983) Osteosarcoma of the jaw. Cancer 51(12): 2311-2316

5. August M, Magenni SP, Dewitt D (1997) Osteogenic sarcoma of the jaws: Factors influencing prognosis. Int $\mathrm{J}$ Oral Maxillofac Surg 26(3):198-204

6. Forteza G, Colmenero B, Lopez-Barea F (1986) Osteogenic sarcoma of the maxilla and mandible. Oral Surg Oral Med Oral Pathol 62(2): 179-184

7. Doval DC, Kumar RV, Kannan V et al. (1997) Osteosarcoma of the jaw bones. Br J Oral Maxillofac Surg 35(5): 357362

8. Finkelstein JB (1970) Osteosarcoma of the jaw bones. Radiol Clin North Am 8(3): 425-443

9. Nakayama E, Sugiura K, Kobayashi I, Oobu K, Ishibashi H, Kanda S (2005) The association between the computed tomography findings, histologic features and outcome of osteosarcoma of the jaw. J Oral Maxillofac Surg 63(3): 311-318

10. Dahlin DC (1978) Bone tumors. 3rd ed. Springfield (IL) Charles E Thomas; $p$ 226-273

11. Mardinger O, Givol N, Talmi YP, et al. (2001) Osteosarcoma of the jaw. The Chaim Sheba Medical Centre Experience. Oral Surg Oral Med Oral Pathol Oral Radiol Endod 91(4): 445451

12. Akpolat N, Yildirim H, Poyraz K (2007) Sacral chondroblastic osteosarcoma misdiagnosed as chondrosarcoma and chordoma. Turk J Med Sci 37(4): $243-$ 249

13. Unni KK (1998) Osteosarcoma of bone. J Orthop Sci 3(5): 287-294

14. Slootweg P, Muller H (1985) Osteosarcoma of the jaw bones: Analysis of 18 cases. J Maxillofac Surg 13(4): $158-166$

15. Winkler K, Bielack SS, Delling G, et al (1993) Treatment of osteosarcoma: Experience of the Co operative osteosarcoma study group (COSS). Cancer Treat Res 62: 269-277

16. Pogrel MA (1988) Inferior hemi maxillectomy for the treatment of palatal tumors. J Oral Maxillofac Surg 46(1): 8587

17. Smeele LE, Kostense PJ, van der waal I, Snow GB (1997) Effect of Chemotherapy on survival of craniofacial osteosarcoma: A systematic review of 201 patients. J Clin Oncol 15(1): 363-367

18. Raymond AK, Spires J, Ayala A, Chawla S, Lee YY, Benjamin RS, et al (1989) Osteosarcoma of head and neck. Lab Invest 60: 76a 